\title{
Modelagem matemática aplicada às capturas das principais lagostas desembarcadas em Pernambuco
}

\author{
Severino Adriano de Oliveira Lima ${ }^{1 *}$ \\ Humber Agrelli Andrade ${ }^{2}$ \\ ${ }^{1}$ Universidade Federal de Rondônia, Departamento de Engenharia de Pesca \\ Rua da Paz, 4.376, Lino Alves Teixeira, CEP 76.916-000, Presidente Médici - RO, Brasil \\ ${ }^{2}$ Universidade Federal Rural de Pernambuco, Departamento de Pesca e Aquicultura \\ Rua Dom Manoel de Medeiros, s/n, Dois Irmãos, CEP 52.171-900, Recife - PE, Brasil \\ * Autor para correspondência \\ limasao@unir.com
}

Submetido em 25/09/2020

Aceito para publicação em 22/02/2021

\section{Resumo}

A extração de lagostas é considerada a atividade pesqueira mais atrativa economicamente na região Nordeste do Brasil. Neste trabalho, foram avaliadas as variações das capturas das lagostas Panulirus meripurpuratus (lagosta vermelha), Panulirus laevicauda (lagosta verde) e Scyllarides brasiliensis (lagosta sapata) no estado de Pernambuco, nos anos de 1999 a 2006, com intuito de verificar tendências nas produções mensais e a relação entre as capturas realizadas nos meses de defeso e na temporada de pesca. As capturas por temporada foram estudadas a partir de modelos exponenciais e análises de correlações. Modelos exponenciais foram significativos com coeficientes de determinação acima de 0,65 para lagosta vermelha em todos os anos e para a lagosta verde a partir de 2002, e descreveram os decaimentos das capturas do início até o final da temporada. A biomassa da lagosta verde mostrou um decaimento mais acentuado do que a da vermelha, possivelmente porque o coeficiente de capturabilidade seria maior. Já para a lagosta sapata, os modelos tiveram valores dos coeficientes de determinação sempre inferiores a 0,40 . A correlação entre a captura legal e ilegal foi significativa apenas para a lagosta verde e apresentou tendência positiva. A magnitude inicial das capturas para a lagosta vermelha com valores abaixo de 25 toneladas no início da temporada de pesca pode ser um indício alarmante, enquanto para lagosta verde, esse valor pode ser de 10 toneladas. Estudos com modelos de excedente de produção são necessários para melhor avaliar os estoques e verificar a hipótese de que a lagosta verde teria um coeficiente de capturabilidade maior que o da lagosta vermelha.

Palavras-chave: Defeso; Lagosta espinhosa; Modelo exponencial; Palinuridae

\section{Abstract}

Mathematical modeling applied to the catches of the main lobsters landed in Pernambuco. Lobster fishing is the most economically attractive activity in Northeast Brazil. In this work, the catch variations of the lobsters Panulirus meripurpuratus (red spiny), Panulirus laevicauda (smoothtail) and Scyllarides brasiliensis (Brazilian slipper lobster) in Pernambuco in the years 1999 to 2006 were evaluated to determine trends in monthly production rates and the relationship between the catches made in the closed period and during open season. Seasonal catches were studied from exponential models and correlation analysis. The exponential models were 
significant and showed determination coefficients above 0.65 for red spiny in all years and for smoothtail starting in 2002, and they described the decline in catches from the beginning to the end of the season. The biomass of the smoothtail showed a more pronounced decline compared to the red lobster, possibly because the capture coefficient would be higher. As for the Brazilian slipper lobster, the models had values of determination coefficients always below 0.40 . The correlation between legal and illegal capture was significant only for smoothtail and showed a positive trend. The initial magnitude of the catch for red spiny below 25 tonnes at the beginning of the fishing season can be an alarming indication, whereas for smoothtail, this value can be 10 tonnes. Studies with surplus production models are necessary to better assess stocks and verify the hypothesis that smoothtail would have a higher capture coefficient than that of red spiny.

Key words: Closed period; Exponential model; Palinuridae; Spiny lobster

\section{Introdução}

As lagostas são megacrustáceos muito apreciados na culinária, tornando sua captura atrativa por ser umas das mais recompensadoras financeiramente em todo o mundo (PHILLIPS et al., 2013). No Brasil, a pesca da lagosta representa um dos recursos pesqueiros mais importantes economicamente (SILVA; FONTELESFILHO, 2011). Há uma diversidade de espécies de lagostas com variadas distribuições no país e, entre elas, as lagostas espinhosas Panulirus meripurpuratus Giraldes e Smyth, 2016 e Panulirus laevicauda (Latreille, 1817) da família Palinuridae são as mais rentáveis (PHILLIPS et al., 2013). Ocorre também destaque para a lagosta Scyllarides brasiliensis Rathbun, 1906, embora essa espécie não seja o principal alvo da captura (LAVALLI; SPANIER, 2007). As três espécies têm distribuição no Atlântico Oeste (GIRALDES; SMYTH, 2016) com sobreposição parcial, principalmente entre $P$. meripurpuratus e $P$. laevicauda (FONTELES-FILHO, 1997).

A história das capturas comercias das lagostas no Brasil tem início na década de 1950 na costa do estado do Ceará (e.g. PAIVA; COSTA, 1963; 1970; FONTELES-FILHO, 2007), enquanto estudos voltados para ecologia, dinâmica populacional e manejo pesqueiro na mesma região tiveram início por volta de 1960 (e.g. PAIVA, 1965; 1966). Em Pernambuco, as capturas iniciaram concomitantemente com o Ceará, no entanto, as informações a respeito da extração no estado não seguiram o mesmo padrão de avaliação. O longo histórico das capturas em Pernambuco não resultou em diversos estudos para as capturas das lagostas, porém há estudos disponíveis dos últimos dados publicados para consulta pública e irrestrita (e.g. LIMA; ANDRADE,
2017). No estado de Pernambuco, a extração de lagostas é considerada a atividade pesqueira mais atrativa economicamente (LESSA et al., 2011) e gerou divisas, para o Brasil, com exportações anuais que superaram US\$ 50.000.000,00 entre os anos de 2000 e 2019 (exceto 2012) e com registros de mais de US\$ $90.000 .000,00 \mathrm{em}$ dois anos (2007 e 2019), tendo como destaque a região Nordeste (MDIC, 2020).

Estudos que ajudem a avaliar o impacto das pescarias sobre as lagostas são preponderantes e, com o aporte deles, medidas de gestão já foram adotadas, como a proibição por tamanho do indivíduo e de alguns apetrechos de pesca, além do período de defeso (SILVA; FONTELES-FILHO, 2011). O período de defeso, que consiste na proibição da captura em determinadas épocas, sendo o principal motivo para a proteção do período reprodutivo, foi adotado para as lagostas como uma medida de gestão não apenas no Brasil, mas também em outros países (e.g. África do Sul, Austrália, Índia, México) (PHILLIPS et al., 2013; RADHAKRISHNAN et al., 2019). Entretanto, essa e outras medidas ainda se mostram insuficientes para a proteção do recurso, que tem apresentado um decréscimo das taxas de captura (IBAMA, 2008). A baixa eficiência do defeso pode estar mais ligada a outras questões, como a ausência de testes para avaliar o fechamento em outras épocas do ano, já que o benefício para o estoque jovem ainda é relativamente pequeno e, principalmente, a falta de fiscalização nesse período (SILVA; FONTELES-FILHO, 2011). No entanto, há indícios de que este tipo de manejo esteja colaborando para preservação do recurso, pois as taxas de captura das lagostas vermelha e verde, por exemplo, são relativamente altas no início da temporada de pesca, quando comparadas com as do final (LIMA; ANDRADE, 2017). 
Adicionalmente, para medidas de gestão, modelos matemáticos visando proteger, otimizar ou mesmo prever as capturas de diversos recursos pesqueiros são amplamente utilizados (e.g. FOX, 1970; HILBORN; WALTERS, 1992), inclusive para as lagostas (e.g. FONTELES-FILHO et al., 1988; CRUZ et al., 2013; ANDRADE, 2015). Esses modelos podem servir de ferramenta para avaliar variações temporais de propriedades relativas ao estoque pesqueiro e estabelecer medidas de gestão. Contudo, para que os resultados dos modelos possam servir de parâmetro é necessária a obtenção de dados confiáveis para viabilizar as estimativas. As principais lagostas capturadas em Pernambuco, de maneira geral, devido à conjuntura econômica das populações isoladas no Brasil, parecem apresentar dados disponíveis mais acurados quando comparadas a várias outras espécies com menor apelo de rentabilidade (LIMA; ANDRADE, 2018). Neste trabalho, foi avaliado o quadro histórico mais recente do qual se tem dados disponíveis para consulta pública e irrestrita das variações das capturas anuais e mensais das lagostas P. meripurpuratus (vermelha), $P$. laevicauda (lagosta verde) e $S$. brasiliensis (lagosta sapata) em Pernambuco. Portanto, o intuito foi, através da modelagem matemática, verificar tendências nas produções mensais e a relação entre as capturas ilegais realizadas nos meses de defeso e as capturas legais na temporada de pesca, contribuindo assim para as ações de gestão dirigidas às lagostas na costa de Pernambuco, polo pioneiro de desenvolvimento da pesca desse recurso na costa do nordeste oriental.

\section{Material e Métodos}

\section{Área de estudo e banco de dados}

A região costeira do estado de Pernambuco tem 21 municípios distribuídos em uma faixa de $187 \mathrm{~km}$, porém foram avaliadas e registradas as capturas das pescarias marítimas e estuarinas para os principais que são, respectivamente, do norte ao sul: Goiana, Ilha de Itamaracá, Itapissuma, Igarassu, Abreu e Lima, Paulista, Olinda, Recife, Jaboatão dos Guararapes, Cabo de Santo Agostinho, Ipojuca, Sirinhaém, Rio Formoso, Tamandaré e São José da Coroa Grande (Figura 1).

FIGURA 1: Municípios litorâneos com desembarques de lagostas avaliados no estado de Pernambuco (adaptado de LIMA; ANDRADE, 2017). GO - Goiana; IT - Itapissuma; II - Ilha de Itamaracá; IG - Igarassu; AL - Abreu e Lima; PA - Paulista; OL - Olinda; RE - Recife; JG - Jaboatão dos Guararapes; CA - Cabo de Santo Agostinho; IP - Ipojuca; SI - Sirinhaém; RF - Rio Formoso; TA - Tamandaré e SJ - São José da Coroa Grande.

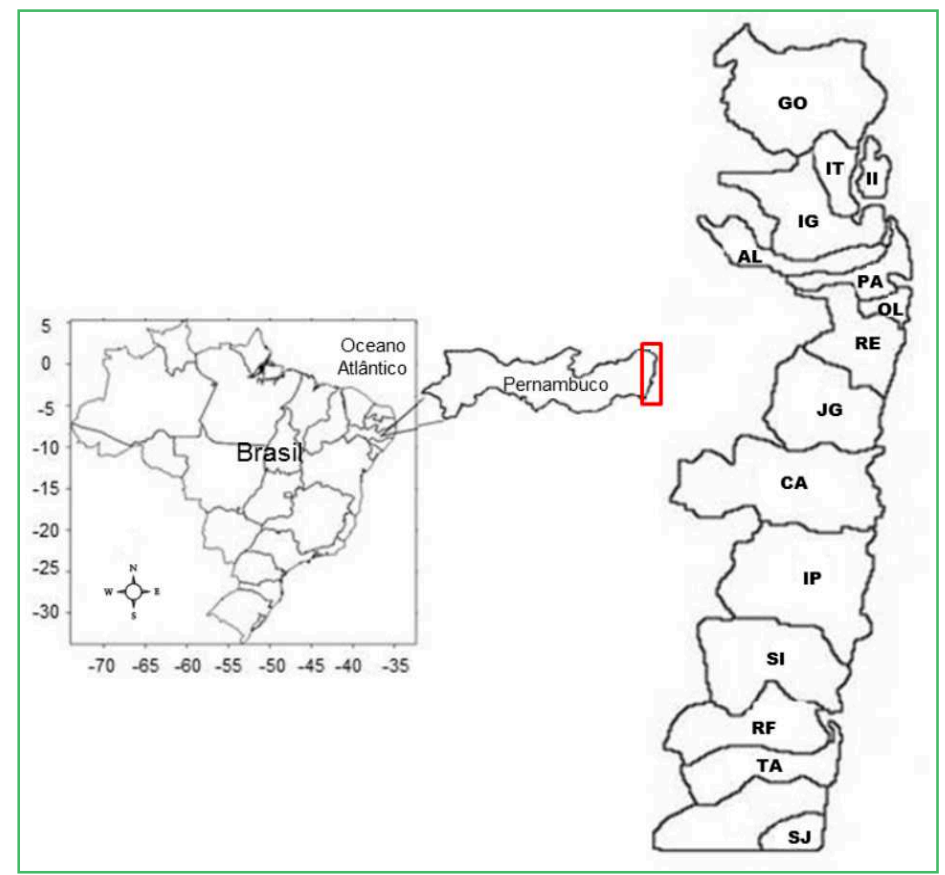


O histórico de captura analisado foi referente aos anos de 1999 a 2006 dos boletins produzidos pelo Centro de Pesquisa e Gestão de Recursos Pesqueiros do Nordeste (CEPENE). As amostragens para produção dos boletins seguiram o sistema de coleta do Programa ESTATPESCA, descrito em Ibama (1994a) e com análise feita por Aragão et al. (2005). Nas fontes de dados, há informações sobre os montantes em peso capturados por mês e por ano, nas pescarias realizadas de forma legalizada ou não das três principais espécies desembarcadas, das lagostas vermelha (P. meripurpuratus), verde ( $P$. laevicauda) e sapata (S. brasiliensis). Ressalta-se que a classificação taxonômica registrada nos boletins para essa primeira foi a espécie Panulirus argus, sendo recente a identificação como outra lagosta distinta, pois $P$. argus tem distribuição no Atlântico Norte até o Caribe, enquanto $P$. meripurpuratus é encontrada na região costeira brasileira (GIRALDES; SMYTH, 2016). No histórico avaliado, o período de defeso ocorreu anualmente entre 1 de janeiro a 30 de abril regulamentado pela Portaria no 137 , de 12 de dezembro de 1994 (IBAMA, 1994b). Capturas realizadas por mergulho eram proibidas no referido período, porém eram efetuadas (IBAMA, 2007; LIMA; ANDRADE, 2017).

\section{Análise dos dados}

Para a realização das análises mensais em todos os anos com os dados disponíveis, considerou-se que a temporada de abertura da pesca teve início no primeiro dia do mês de maio e que o término da temporada se dá no último dia do mês de dezembro. As capturas por temporada foram estudadas a partir de gráficos e de modelos exponenciais do tipo, em que $y$ é a captura em toneladas, $\alpha$ e $\beta$ são parâmetros a serem estimados e $x$ corresponde ao tempo decorrido desde o início da temporada, medido em meses. No modelo exponencial $\mathrm{dy} / \mathrm{dx}=\mathrm{y}$.beta, a taxa de variação da captura depende da captura do momento beta $=1 / \mathrm{y} d y / d x$. Para simplificação da interpretação das estimativas dos parâmetros, os meses são representados pela variável numérica $x$, com a origem da escala $x=0$ representando o mês de maio (abertura da temporada). Assim $x=1$ representa junho e assim por diante até $x=7$ para dezembro. Com essa escala, a estimativa de $\alpha$, que corresponde ao intercepto no eixo $y$, é um índice da magnitude da captura no início da temporada. A estimativa de $\beta$ é também de particular interesse. Valores positivos indicam capturas mensais maiores no decorrer da temporada, e o contrário ocorre caso o valor seja negativo. Para entendimento e interpretação de variações de beta é importante notar que se assume usualmente que y $=\mathrm{qfB}$, em que q é o coeficiente de capturabilidade, f é o esforço de pesca e B é a biomassa. Assim, beta: $\mathrm{y}=\mathrm{dy} / \mathrm{dx}=\mathrm{qfdB} / \mathrm{dx}+\mathrm{qBdf} /$ $\mathrm{dx}+\mathrm{fBdq} / \mathrm{dx}$. Então a taxa de variação da captura ao longo do tempo reflete variações em uma ou mais das três quantidades q, f e B.

No ajuste do modelo aos dados foi utilizada a forma linearizada da equação exponencial, ou seja, . Essa abordagem é conveniente no sentido de que a regressão linear simples pode ser utilizada para calcular as estimativas dos parâmetros, as variâncias e os erros padrão. A inclinação da reta representa então a taxa de variação da captura (em escala logarítmica) em função do tempo $(x)$. Quanto maior o módulo do parâmetro $\beta$, maior será a taxa de variação de $\log (\mathrm{y})$ em função de $\mathrm{x}$.

Para avaliar se as regressões lineares são significantes, foram realizados testes de hipóteses de $t$-Student para a inclinação $(\beta)$. Somente foram explorados os casos em que os coeficientes de determinação $\left(\mathrm{R}^{2}\right)$ são elevados e as inclinações significativamente diferentes de zero. Embora a estimativa da inclinação para a forma linear corresponda diretamente à estimativa de $\beta$ da forma não linearizada, este parâmetro mede a taxa de variação da captura por unidade de captura em cada instante . Por outro lado, o intercepto corresponde ao logaritmo de $\alpha$, e faz-se necessário calcular estimativa do parâmetro e do erro padrão de $\alpha$. Aproximações dessas estimativas foram obtidas com o método delta, baseado na expansão de Taylor (SEBER, 1982).

Análises entre as capturas legais, que correspondem ao somatório entre os meses de maio e dezembro (sem defeso), e as capturas ilegais, que são as capturas somadas entre janeiro e abril (com defeso), nos anos de 1999 a 2006, foram efetuadas para avaliar possíveis correlações entre as duas variáveis, através de testes não paramétricos de correlação de Spearman $(\alpha=0,05)$. Foi utilizado o cálculo do coeficiente de correlação $(r)$, pois 
os pressupostos (normalidade e homocedasticidade) não foram atendidos. Todas as análises foram realizadas com o programa R 4.0.0 (R CORE TEAM, 2020).

\section{Resultados}

Ao ajustar as formas linearizadas dos modelos exponenciais para a captura em período correspondente à temporada de pesca (maio a dezembro), nos anos de 1999 a 2005, observou-se que no caso da lagosta vermelha, no ano de 1999, o modelo regressão explicou $70 \%$ da variação na captura com o tempo decorrido desde o início da temporada (Figura 2A). A qualidade do ajuste aumenta nos anos seguintes, com valores de $\mathrm{R}^{2}$ superiores a 0,70 . No caso da lagosta verde, o modelo de regressão linear explicou menos de $40 \%$ da variação na captura, nos anos de 1999 a 2001. A partir do ano de 2002, os coeficientes de determinação tiveram valores acima de 0,65 (Figuras 2B). Já no caso da lagosta sapata, os coeficientes de determinação dos modelos de regressão linear explicaram menos de $40 \%$ da variação na captura em todos os anos (Figura 2C).

Os gráficos com ajustes dos modelos exponenciais para as lagostas vermelha, verde e sapata são apresentados nos anos que tiveram os maiores coeficientes de determinação, sendo em 2002, 2005 e 2001, respectivamente (Figura 3). No caso da lagosta vermelha (Figura 3A), nota-se que o modelo exponencial

FIGURA 2: Coeficientes de determinação $\left(\mathrm{R}^{2}\right)$ para os ajustes das formas linearizadas dos modelos exponenciais aos dados de captura das lagostas vermelha (A), verde (B) e sapata (C). Os casos em que as inclinações foram significativamente diferentes de zero são indicados por círculos preenchidos.

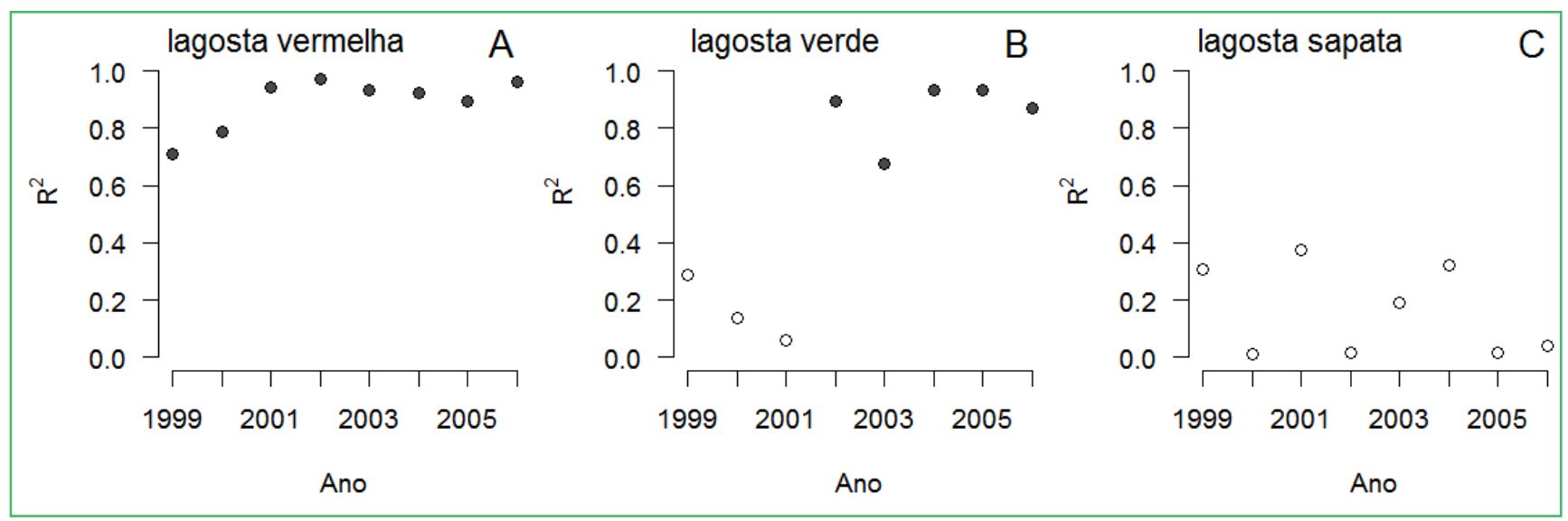

FIGURA 3: Ajuste do modelo exponencial para as capturas de lagosta vermelha na temporada de 2002 (A), de lagosta verde na temporada de 2005 (B) e de lagosta sapata na temporada de 2001 (C). Círculos não preenchidos indicam meses de defeso e os preenchidos meses em que ocorrem capturas legais.

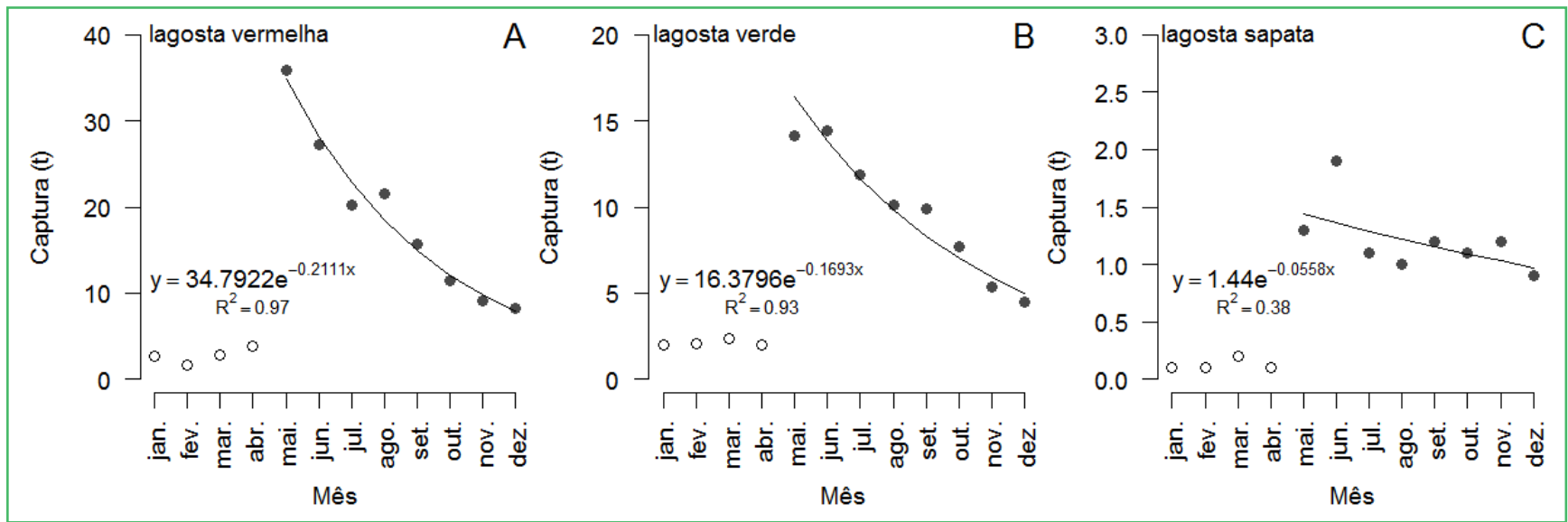


$\left(\mathrm{R}^{2}=0,97\right)$ descreveu o decaimento das capturas desde a abertura até o final da temporada. No caso da lagosta verde (Figura 3B), o modelo $\left(\mathrm{R}^{2}=0,93\right)$ também apresentou tendência de decaimento das capturas, mas este começa a ocorrer somente a partir de julho, no terceiro mês após a abertura da temporada. No caso da lagosta sapata (Figura 3C), mesmo com o modelo de maior coeficiente de determinação $\left(\mathrm{R}^{2}=0,38\right)$, não ocorreu padrão de decaimento acentuado, pois capturas relativamente elevadas aparecem já no segundo mês logo após a abertura da temporada (junho), assim o decaimento das capturas é muito suave, o que resulta em não rejeição da hipótese de que a inclinação é igual a zero $(p=0,11)$. Ainda, as estimativas da inclinação de todas as temporadas não indicaram diferenças significativas de zero porque a declividade das capturas foi sempre inexistente ou muito suave, como mostrado no exemplo da Figura 3C.
Na Figura 4 são mostradas as estimativas dos parâmetros e os respectivos intervalos de confiança para os casos em que o ajuste do modelo exponencial se mostra adequado, no sentido de que as estimativas das inclinações e dos coeficientes de determinação são significativamente diferentes de zero. Para a lagosta vermelha, as estimativas da magnitude inicial das capturas $(\alpha)$ variaram bastante entre os anos, mas não apresentaram tendências de declínio ou incremento, e na maioria dos casos foram superiores a 30 toneladas (Figura $4 \mathrm{~A})$. As inclinações ( $\beta$ ) variaram acentuadamente nos primeiros anos da série temporal com uma queda de - 0,1 para - 0,3 de 2000 a 2001 (Figura 4B). Posteriormente, há uma tendência de que as inclinações se tornem progressivamente mais suaves (Figura 4B) e, de maneira geral, se mantêm entre - 0,2 e - 0,1 a partir de 2003. No caso da lagosta verde, as estimativas de $\alpha$ são todas bem menores (cerca de 15 toneladas) que as estimativas para

FIGURA 4: Estimativas dos parâmetros dos modelos exponenciais e respectivos intervalos de confiança (95\%) marginais.

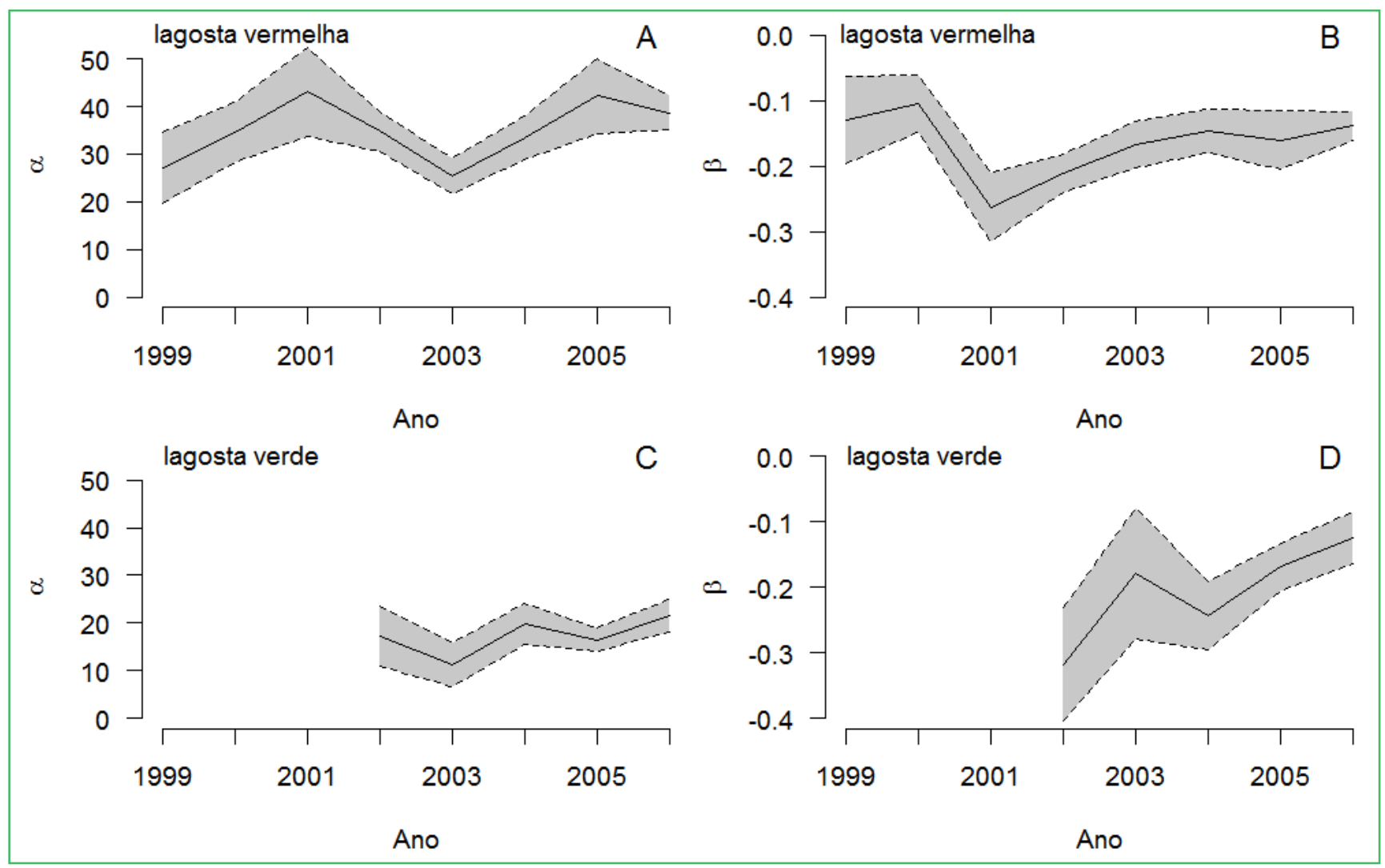


a lagosta vermelha. No entanto, há uma leve tendência de incremento no decorrer dos anos (Figura 4C). As estimativas da inclinação $(\beta)$ para a lagosta verde apresentam intervalos de confiança relativamente mais amplos. Nota-se também que elas são relativamente reduzidas (inferiores a - 0,3) especialmente em anos anteriores a 2005 (Figura 4D). De maneira geral, há uma tendência de que as inclinações se tornem mais suaves a partir de 2004.

As tendências das correlações entre as capturas legais e ilegais foram positivas para as lagostas vermelha e verde (Figuras 5A e 5B), enquanto para a lagosta sapata (Figura 5C) foi negativa. Os coeficientes de correlação foram menores para as lagostas vermelha e lagosta sapata quando comparados aos da lagosta verde, sendo que para esta ocorreu correlação significativa $(S=15, p=0,01)$, mas não para as lagostas vermelha $(S=56, p=0,43)$ e sapata $(S=128, p=0,18)$. Considerando o módulo dos valores do coeficiente angular, a lagosta verde foi a que teve o maior valor, em seguida a sapata e por último a vermelha, ou seja, um valor maior da produção legal anual denota que a produção ilegal para a lagosta verde neste mesmo ano seria mais acentuada.

\section{Discussão}

As estimativas das inclinações $(\beta)$ dos modelos exponenciais no geral indicaram decaimentos ao longo dos meses durante os anos analisados mais acentuados no caso da lagosta verde, o que é especialmente evidente entre 2002 e 2004. A exploração das diferenças dos valores de $\beta$ calculados para as lagostas verde e vermelha depende do entendimento do que esse parâmetro representa e dos fatores que podem afetar o seu valor. Como já mencionado, $\beta$ é a taxa de variação da captura (em escala logarítmica) em função do tempo. Como todos os valores estimados para $\beta$ são negativos, a variação da captura, como esperado, é maior no início e menor no final do período avaliado. Assim, $\beta$ representa a taxa de decaimento da captura em peso $(Y)$. De maneira simplificada, a variável $Y$ pode ser vista como função de combinações da biomassa $(B)$, do coeficiente de capturabilidade $(q)$ e do esforço de pesca $(f)$, ou do coeficiente de mortalidade por pesca $(F)$, na forma $Y=q \times f \times B=F \times B$. Portanto, a inclinação $\beta$ será um valor elevado em módulo se no decorrer da temporada o coeficiente de capturabilidade $(q)$, ou o esforço de pesca $(f)$, ou biomassa $(B)$, ou duas destas quantidades, ou todas elas, reduzirem muito no decorrer da temporada. Nos boletins estatísticos governamentais não há informações suficientes para conduzir análises que pudessem ajudar a discernir entre os efeitos das três principais covariáveis envolvidas. No entanto, no caso da comparação das declividades $(\beta)$ das capturas da lagosta verde e da vermelha, os esforços $(f)$ são sempre os mesmos para as duas espécies, já que as capturas de lagosta no Brasil são multiespecíficas,

FIGURA 5: Estimativas das regressões lineares e coeficientes de determinação para os dados de captura anuais das lagostas vermelha (A), verde (B) e sapata (C) obtidos nos anos de 1999 a 2006.

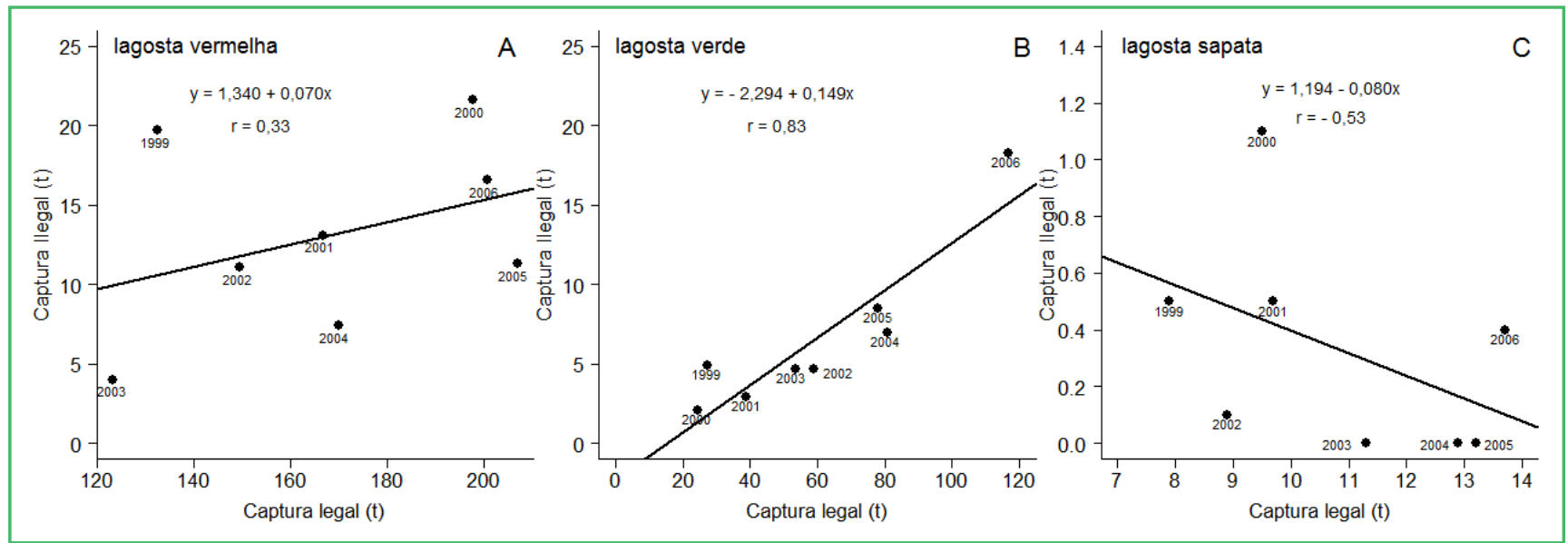


produzindo quantidades variadas de lagosta vermelha e verde, usando o mesmo equipamento e unidade de esforço (CRUZ et al., 2013). Portanto, as diferenças nos valores de $\beta$ das duas espécies é decorrente somente de modificações em somente dois fatores, $q$ e $B$. Ocorreria então pelo menos duas possibilidades: a) o coeficiente de capturabilidade $(q)$ da lagosta verde é no geral mais alto que o da vermelha, o que provocaria uma mortalidade por pesca $(F)$ que levaria a uma redução mais acentuada da biomassa; ou b) os valores de $q$ poderiam decair mais rapidamente para a lagosta verde do que para a vermelha no decorrer da temporada, ainda que a biomassa desse recurso não diminuísse tão rápido quanto o da lagosta vermelha.

Uma diminuição acentuada de $q$ pode ocorrer no período de maior atividade reprodutiva, já que as fêmeas apresentam então menor atividade trófica e estão menos propensas a serem atraídas pela isca e capturadas (PAIVA; COSTA, 1964). No entanto, na revisão de Ivo e Pereira (1996), menciona-se que os picos de maior atividade reprodutiva das lagostas verde e vermelha seriam coincidentes, iniciando em janeiro e estendendo-se até julho, porém essa informação tem que ser considerada de forma cautelosa, principalmente por causa dos impactos climáticos que podem ocasionar mudanças comportamentais (e.g. distribuição) nas lagostas (BOAVIDA-PORTUGAL et al., 2018). Com a permanência do padrão reprodutivo, os referidos autores citaram ainda que haveria um segundo pico menos pronunciado no segundo semestre do ano, para o qual não há inclusive registros importantes de fêmeas da lagosta verde com ovos. Em suma, não há elementos que indiquem claramente que a dinâmica reprodutiva diferenciada apontaria que o decaimento do coeficiente de capturabilidade no decorrer da temporada seja mais acentuado para a lagosta verde do que para a lagosta vermelha. Portanto, ganha força a alternativa de que a biomassa da lagosta verde decai mais rapidamente no decorrer da temporada porque o coeficiente de capturabilidade dessa espécie seria maior que o da lagosta vermelha. No que diz respeito ao efeito da distribuição espacial, de fato, é provado que a população de lagosta verde tem distribuição proporcionalmente mais costeira (PAIVA, 1967; IVO; PEREIRA, 1996) e sofreria mais fortemente o efeito do esforço de pesca. Baseado em análises das distribuições espaciais do esforço de pesca e das taxas de captura da frota que operou com armadilhas entre os anos de 1974 e 1989, Fonteles-Filho (1997) similarmente chegou à conclusão de que a lagosta verde estaria mais sujeita à sobre-exploração do que a lagosta vermelha.

Estudos de avaliação de estoques com o ajuste de modelos que têm o coeficiente de capturabilidade $(q)$ como parâmetro (e.g. modelos de excedente de produção) são necessários para se tirar uma conclusão acerca da hipótese de que a lagosta verde teria um $q$ maior que o da lagosta vermelha. A abundância menor da lagosta verde (FONTELES-FILHO, 1997) explicaria então as estimativas de $\alpha$ (captura esperada para os inícios das temporadas) sistematicamente menores mesmo que os coeficientes de capturabilidade sejam então em geral maiores. Assim, chama particular atenção o risco da lagosta verde diante da exploração pesqueira, pois ela seria mais vulnerável (maior $q$ ) e menos abundante (menor $B$ ) que a vermelha (FONTELES-FILHO, 1992; 1997). Essa combinação de fatores colocaria a lagosta verde em um patamar de maior fragilidade frente ao esforço de pesca.

Há diferenças importantes entre as estimativas dos parâmetros para as duas espécies (lagosta vermelha e lagosta verde), mas há também diferenças importantes entre as temporadas de pesca para uma mesma espécie. Destacam-se, por exemplo, as variações das inclinações $(\beta)$, que, no caso da lagosta vermelha, se apresenta suave em 1999 e 2000, acentuada em 2001 e posteriormente cada vez menos acentuadas até 2006. O padrão de diminuição (em módulo) da inclinação a partir de 2001 é também evidente no caso da lagosta verde. Como $Y=$ $F \times B$, o que faria a captura $(Y)$ cair mais lentamente no decorrer dos anos está atrelado a $F$ ou $B$. Assim, há pelo menos duas hipóteses para o caso: a) ao longo dos anos ocorre uma gradativa tendência de que a mortalidade por pesca $(F)$ seja intensificada cada vez mais no decorrer da temporada, de tal forma que as capturas decaiam cada vez mais lentamente ao longo dos meses mesmo que as biomassas diminuam; ou b) as biomassas decairiam mais lentamente no decorrer das temporadas dos anos da década de 2000. Assim, as capturas também decairiam mais lentamente para níveis de mortalidade por pescas 
similares. Investigações sobre índices da abundância (e.g. B) e/ou a obtenção de informações que permitam o ajuste de modelos em que explicitamente se estima a mortalidade por pesca ou a biomassa são necessárias para esclarecer a questão.

De maneira geral, há tendências temporais não claras na variação anual da captura esperada $(\alpha)$ no início das temporadas. Em um primeiro momento percebese que as medidas de manejo adotadas (e.g. defeso e tamanhos mínimos) têm sido o suficiente para manter o mesmo nível de rendimento nos últimos anos. No entanto, é importante mencionar que, na ausência de informações sobre as variações interanuais do esforço das diversas modalidades de pesca (i.e. armadilha e rede de emalhe) ou mesmo de estimativas de taxas de captura (e.g. captura por unidade de esforço - CPUE), seria precipitado tirar qualquer conclusão de que há uma estabilidade no suprimento de biomassa de lagosta para a costa e a plataforma continental de Pernambuco. Infelizmente, a lacuna de informações de pesca existente a partir do final da década de 1990 transcende Pernambuco e é uma realidade para a pesca da lagosta no Nordeste como um todo. As séries temporais de esforço de pesca e CPUE estão disponíveis somente para anos anteriores a 1999 (e.g. SILVA; FONTELESFILHO, 2011).

As correlações para as lagostas vermelha e sapata não foram significativas, porém pode-se verificar que os padrões apontados para cada uma delas são opostos: enquanto para a primeira uma taxa maior de captura anual ilegal pode ser um reflexo de uma maior abundância nas produções legais e vice-versa, na lagosta sapata a taxa de captura ilegal anual já compromete o estoque e consequentemente diminui a produção legal do ano. As tendências distintas para essas espécies podem estar ligadas ao tamanho de suas populações, pois a lagosta vermelha, entre as lagostas na costa brasileira, é a que tem a maior parte das capturas e estoque, já as capturas para lagosta sapata são pouco expressivas (IBAMA, 2008; MPA, 2012; CRUZ et al., 2013). O resultado da inclinação positiva para lagosta verde é considerado o mais importante devido a sua significância estatística, adicionalmente, essa é uma espécie que tem distribuição quase que restrita à costa brasileira, sendo o país o maior produtor global (BAISRE; ALFONSE, 1994). Assim os resultados obtidos neste trabalho podem servir de parâmetro para futuras avaliações das capturas ilegais, que possivelmente ainda continuam sendo realizadas em Pernambuco.

Embora as medidas de gestão aplicadas nas pescarias das lagostas não tenham surtido o efeito esperado desde a década de 1960, a ineficiência de tais medidas decorreu principalmente de diversas normas (e.g. tamanho mínimo de captura, limitação dos desembarques e proibição da captura de jovens ou fêmeas ovadas) aplicadas concomitantemente, o que dificultou ainda mais o processo de fiscalização, que sempre foi deficitário (DIAS NETO, 2003; CAVALCANTE et al., 2011). Porém, entre as medidas existentes, é inegável que o defeso tem contribuído, funcionando como elemento de acumulação de abundância, e os benefícios desse tipo de manejo podem ser melhorados com maior eficiência da fiscalização e considerando fatores biológicos (como a proteção de fêmeas ovígeras), econômicos (com o aumento do esforço nas épocas em que a produtividade do estoque é mais elevada) e espaciais (com um defeso para proteger os juvenis em seus criadouros) (SILVA; FONTELES-FILHO, 2011; CRUZ et al., 2013).

Os dados das capturas para as lagostas em Pernambuco nessa série histórica analisada podem refletir também outros fatores, como dinâmica da frota $\mathrm{e}$ mudanças da arte de pesca (LIMA; ANDRADE, 2017). Por isso é prudente que o uso dos resultados dos modelos seja reavaliado em estudos futuros com uma série de dados mais longa e que sejam consideradas outras covariáveis, como as citadas anteriormente. No entanto, de acordo com os resultados apresentados, a magnitude inicial das capturas $(\alpha)$ para a lagosta vermelha com valores abaixo de 25 toneladas no início da temporada de pesca pode ser um indício alarmante. No caso da lagosta verde, com a maior estabilização a partir de 2002, um valor de $\alpha$ menor que 10 toneladas pode também significar um problema como, por exemplo, sobre-exploração em anos anteriores. As estimativas das inclinações $(\beta)$ para lagosta verde foram maiores, indicando decaimentos das capturas mais acentuado ao longo dos meses nos anos avaliados, portanto estudos 
como modelos de excedente de produção são necessários para melhor avaliar os estoques e comparar, por exemplo, se a lagosta verde teria um coeficiente de capturabilidade maior que o da lagosta vermelha.

\section{Agradecimentos}

À Coordenação de Aperfeiçoamento de Pessoal de Nível Superior (CAPES), por conceder uma bolsa de estudo de pós-graduação no momento da pesquisa ao primeiro autor.

\section{Referências}

ANDRADE, H. A. Stock assessment of the red spiny lobster (Panulirus argus) caught in the tropical southwestern Atlantic. Latin American Journal of Aquatic Research, Valparaíso, v. 43, n. 1, p. 201-214, 2015.

ARAGÃO, J. A. N.; ROCHA, C. A. S. R.; PETRERE JUNIOR, M. Análise da consistência estatística do programa de coleta de dados de desembarque de pescado, executado pelo IBAMA no nordeste do Brasil. Boletim Técnico Científico do CEPENE, Tamandaré, v. 13, n. 2, p. 97-127, 2005.

BAISRE, J. A.; ALFONSE, I. Late stage larvae of Panulirus guttatus (Latreille, 1804) (Decapoda, Palinuridae) with notes on the identification of phyllosoma of Panulirus in the Caribbean Sea. Crustaceana, Leiden, v. 6, n. 1, p. 25-34, 1994.

BOAVIDA-PORTUGAL, J.; ROSA, R.; CALADO, R.; PINTO, M.; BOAVIDA-PORTUGAL, I.; ARAÚJO, M. B.; GUILHAUMON F. Climate change impacts on the distribution of coastal lobsters. Marine Biology, Kiel, v. 2018, p. 165-186, 2018.

CAVALCANTE, P. P. L.; FURTADO-NETO, A. M. A.; COSTA, J. M.; NEVES, S. S. Ordenamento da pesca da lagosta: uma experiência desordenada. Fortaleza: MMA e UFC, 2011. 253 p.

CRUZ, R. A.; SILVA, K. C. A.; CINTRA, I. Assessment of wild spiny lobster stocks on the Brazilian continental shelf. Crustaceana, Leiden, v. 86, n. 3, p. 336-356, 2013.

DIAS NETO, J. Gestão do uso dos recursos pesqueiros marinhos no Brasil. Brasília: MMA, 2003. 242 p.

FONTELES-FILHO, A. A. Population dynamics of spiny lobsters (Crustacea: Palinuridae) in northeast Brazil. Ciência e Cultura, São Paulo, v. 44, n. 2, p. 192-196, 1992.

FONTELES-FILHO, A. A. Spatial distribution of the lobster species Panulirus argus and P. laevicauda in northern and northeastern Brazil in relation to the distribution of fishing effort. Ciência e Cultura, São Paulo, v. 49, n. 3, p. 172-176, 1997.

FONTELES-FILHO, A. A. Síntese sobre a lagosta-vermelha (Panulirus argus) e a lagosta-verdade (Panulirus laevicauda) do Nordeste do Brasil. In: HAIMOVICI, M. (Ed.). A prospecção pesqueira e abundância de estoques marinhos no Brasil nas décadas de 1960 a 1990: levantamento de dados e avaliação crítica. Brasília: Ministério do Meio Ambiente, 2007. p. 257-265.
FONTELES-FILHO, A. A.; XIMENES, M. O.; MONTEIRO, P. H. Sinopse de informações sobre as lagostas Panulirus argus (Latreille) e Panulirus laevicauda (Latreille) (Crustacea: Paniluridae), no nordeste do Brasil. Arquivos de Ciências do Mar, Fortaleza, v. 27, n 1, p. 1-19, 1988.

FOX, W. W. An exponential yield model for optimizing exploited fish populations. Transactions of the American Fisheries Society, Oxford, v. 99, n. 1, p. 80-88, 1970.

GIRALDES, B. W.; SMYTH, D. M. Recognizing Panulirus meripurpuratus sp. nov. (Decapoda: Palinuridae) in Brazil Systematic and biogeographic overview of Panulirus species in the Atlantic Ocean. Zootaxa, Auckland, v. 4107, n. 3, p. 353-366, 2016.

HILBORN, R.; WALTERS, C. J. Quantitative fisheries stock assessment. New York: Chapman \& Hall, 1992. 570 p.

IBAMA. Manual do Estatpesca. Fortaleza: MMA, 1994a. 57 p.

IBAMA. Portaria no 137, de 12 de dezembro de 1994. 1994b.

IBAMA. Estatística da Pesca 2007 - Brasil - Grandes Regiões e Unidades da Federação. Brasília: MMA, 2007. 113 p.

IBAMA. Plano de gestão para o uso sustentável de lagostas no Brasil: Panulirus argus (Latreille, 1804) e Panulirus laevicauda (Latreille, 1817). Brasília: MMA, 2008. 121 p.

IVO, C. T. C.; PEREIRA, J. A. Sinopse das principais observações sobre as lagostas Panulirus argus (Latreille) e Panulirus laevicauda (Latreille), capturadas em águas costeiras do Brasil, entre os estados do Amapá e do Espírito Santo. Boletim Técnico Científico do CEPENE, Tamandaré, v. 4, n. 1, p. 7-94, 1996.

LAVALLI, K. L.; SPANIER, E. Introduction to the biology and fisheries of Slipper Lobsters, Chapter 1. In: LAVALLI, K. L.; SPANIER, E. (Ed.). The Biology and Fisheries of the Slipper Lobster. Boca Raton: Taylor e Francis Group, Crustacean Issues, CRC Press, 2007. p. 3-21.

LESSA, R. P.; MONTEIRO, A.; DUARTE-NETO, P. J.; VIEIRA, A. C. Análise multidimensional dos sistemas de produção pesqueira do estado de Pernambuco, Brasil. In: HAIMOVICI, M. (Ed.). Sistemas pesqueiros marinhos e estuarinos do Brasil. Rio grande do Sul: Editora da FURG, 2011. p. 41-54.

LIMA, S. A. O.; ANDRADE, H. A. Variações nas capturas das lagostas vermelha (Panulirus meripurpuratus), verde (Panulirus laevicauda Latreille, 1817) e sapata (Scyllarides brasiliensis Rathbun, 1906) na costa de Pernambuco. Boletim do Instituto de Pesca, São Paulo, v. 43, n. 2, p. 194-206, 2017.

LIMA, S. A. O.; ANDRADE, L. H. A. Análise da evolução do setor pesqueiro de Pernambuco. Arquivos de Ciências do Mar, Fortaleza, v. 51, n. 2, p. 27-43, 2018.

MDIC. Sistema de Análise das Informações de Comércio Exterior - Comex Stat. 2020. Disponível em <http: www. comexstat.mdic.gov.br/pt/home>.

MPA. Boletim estatístico da pesca e aquicultura. Brasília: MPA, 2012. 129 p.

PAIVA, M. P. Dinâmica da pesca de lagostas no Ceará. Arquivos de Ciências do Mar, Fortaleza, v. 5, n. 2, p. 151-174, 1965.

PAIVA, M. P. Estudo sobre a pesca de lagostas no Ceará no ano de 1965. Arquivos de Ciências do Mar, Fortaleza, v. 6, n. 2, p. $147-165,1966$. 
PAIVA, M. P. Algunos problemas de la industria langostera em el Brasil. Arquivos de Ciências do Mar, Fortaleza, v. 7, n. 2, p. $105-$ 112, 1967.

PAIVA, M. P.; COSTA, R. S. Estudos de biologia da pesca de lagosta no Ceará - Dados de 1962. Arquivos de Ciências do Mar, Fortaleza, v. 3, n. 1, p. 27-52, 1963.

PAIVA, M. P; COSTA, R. S. Estudos de biologia da pesca de lagostas no Ceará - dados de 1963. Arquivos de Ciências do Mar, Fortaleza, v. 4, n. 2, p. 45-70, 1964.

PAIVA, M. P.; COSTA, R. S. Estudos de biologia da pesca de lagosta no Ceará - Dados de 1969. Arquivos de Ciências do Mar, Fortaleza, v. 10, n. 2, p. 131-142, 1970.
PHILLIPS, B. F.; MELVILLE-SMITH, R.; KAY, M. C.; VEGAVELÁZQUEZ, A. Panulirus species. In: PHILLIPS, B. F. (Ed.). Lobsters: biology, management, aquaculture, and fisheries. Oxford: Wiley-Blackwell, 2013. p. 289-325.

R CORE TEAM. R: a language and environment for statistical computing. 2020. Disponível em <https://www.R-project.org/>.

RADHAKRISHNAN, E. V.; PHILLIPS, B. F.; ACHAMVEETIL, G. Lobsters: biology, fisheries and aquaculture. Singapore: Springer, 2019. $683 \mathrm{p}$.

SEBER, G. A. F. The estimation of animal abundance. London: Charles Griffin, 1982. 672 p.

SILVA, A. C.; FONTELES-FILHO, A. A. Avaliação do defeso aplicado à pesca da lagosta no nordeste do Brasil. Fortaleza: Editora Expressão Gráfica, 2011. 110 p. 